Article

\title{
Magnetorheological Elastomer-Based Self-Powered Triboelectric Nanosensor for Monitoring Magnetic Field
}

\author{
Dong Wan ${ }^{1, \dagger}$, Ningchen Ma ${ }^{2,+}$, Taochuang Zhao ${ }^{1,+}$, Xiaojing Cui ${ }^{1}$, Zhaosu Wang ${ }^{1}$, Hulin Zhang ${ }^{1}$ \\ and Kai Zhuo ${ }^{1, *}$ \\ 1 College of Information and Computer, Taiyuan University of Technology, Taiyuan 030024, China; \\ 18771046115@163.com (D.W.); ztttry@163.com (T.Z.); cui85851328@163.com (X.C.); \\ 15841531501@163.com (Z.W.); zhanghulin@tyut.edu.cn (H.Z.) \\ 2 AIEN Institute, Shanghai Ocean University, Shanghai 201306, China; mnc425@126.com \\ * Correspondence: zhuokai@tyut.edu.cn \\ + Dong Wan, Ningchen Ma and Taochuang Zhao contributed equally to the work.
}

check for

updates

Citation: Wan, D.; Ma, N.; Zhao, T.; Cui, X.; Wang, Z.; Zhang, H.; Zhuo, K. Magnetorheological Elastomer-Based Self-Powered Triboelectric

Nanosensor for Monitoring Magnetic Field. Nanomaterials 2021, 11, 2815.

https://doi.org/10.3390/

nano11112815

Academic Editors: Julian Maria

Gonzalez Estevez and

Régine Perzynski

Received: 22 September 2021

Accepted: 21 October 2021

Published: 23 October 2021

Publisher's Note: MDPI stays neutral with regard to jurisdictional claims in published maps and institutional affiliations.

\begin{abstract}
The adaptable monitoring of the ubiquitous magnetic field is of great importance not only for scientific research but also for industrial production. However, the current detecting techniques are unwieldly and lack essential mobility owing to the complex configuration and indispensability of the power source. Here, we have constructed a self-powered magnetic sensor based on a subtle triboelectric nanogenerator (TENG) that consists of a magnetorheological elastomer (MRE). This magnetic sensor relies on triboelectrification and electrostatic induction to produce electrical signals in response to the MRE's deformation induced by the variational magnetic field without using any external power sources. The fabricated magnetic sensor shows a fast response of $80 \mathrm{~ms}$ and a desirable sensitivity of $31.6 \mathrm{mV} / \mathrm{mT}$ in a magnetic field range of 35-60 mT as well as preliminary vectorability enabled by the multichannel layout. Our work provides a new route for monitoring dynamic magnetic fields and paves a way for self-powered electric-magnetic coupled applications.
\end{abstract}

Keywords: self-powered; triboelectric; magnetorheological elastomer; magnetic

\section{Introduction}

The magnetic field is a vector quantity, which means that it has both magnitude and direction. For many years, researchers have been studying the application of magnetic fields [1-9], and have found that they play a significant role in geophysics, space technology, medical applications, and other areas [10-13]. Currently, there are many methods used to measure magnetic fields, such as magnetic force, hall effect, fluxgate, magnetic resonance, and superconductivity effects. Based on these methods, different principles of using magnetic field measurement instruments have been implemented, and all of these techniques for measuring magnetic fields have their benefits (Table S1) [14]. However, all of the above-mentioned measuring instruments require an external power supply, so it is urgent to develop a self-powered magnetic field monitoring instrument.

MRE is a magnetically sensitive smart composite material prepared from ferromagnetic particles and a polymer-like matrix, whose material properties can be quickly and reversibly controlled by an external magnetic field [15-21]. Because the matrix of MRE is a solid polymer material, the performance of MRE is simultaneously stable and reproducible. In recent years, a large number of researchers have started to focus on self-powered technologies [22-26]. Among them is TENG: an emerging technology that can directly convert mechanical energy into electrical energy [24,27-32]. Since TENG has a high sensitivity to mechanical triggering, many self-powered sensors based on TENG have been designed [16,24,33-38]. Here, we designed a self-powered magnetic field monitoring sensor. 
In this work, we used styrene ethylene butylene styrene (SEBS) as a substrate and added magnetic fluid to prepare MRE films [39]. In order to measure the strength and direction of the magnetic field, a self-powered sensor based on the MRE of TENG is reported in this paper. The structure and mechanism of the MRE-based TENG are described here in detail, and the material properties of the MRE and the output signals of the sensor are systematically investigated. With the assistance of a programmable platform, the newly designed pointer-based TENG structure enables excellent real-time magnetic field monitoring and unique self-powered capabilities. This work promotes the use of TENG-based sensing applications for magnetic field measurement, which has important implications for IoT, robotics, and AI.

\section{Discussion and Results}

The structural design of the MRE-based TENG device is illustrated in Figure 1a. When a magnetic field perpendicular to the TENG is applied, the MRE film deforms and drives the PTFE close to the $\mathrm{Al}$ under the action of the magnetic field. At the right bottom, a photograph of the fabricated TENG is presented, which shows how the PTFE is conformally pasted on the MRE film. As illustrated in Figure 1b, the scanning electron microscopy (SEM) image of the MRE film shows that the ferruginous particles were uniformly distributed in the SEBS elastomer; this is verified via an EDS test plotted in Figure 1c. The emerging Fe originated from the mixed ferruginous powder, with the observed $\mathrm{C}$ and $\mathrm{O}$ elements resulting from the SEBS polymer. This is the same as the expected XRD result, indicating that the prepared film consists of $\mathrm{Fe}_{3} \mathrm{O}_{4}$ nanoparticles. As shown in Figure 1d, the diffraction peaks were concentrated at $30.6^{\circ}, 35.8^{\circ}, 45.2^{\circ}$, $63.01^{\circ}$, and $70.2^{\circ}$, and are consistent with the face-centered cubic $\mathrm{Fe}_{3} \mathrm{O}_{4}$ (JCPDS No. 190629). Figure 1e shows the hysteresis loop of the SEBS and MRE in the range of $-10 \mathrm{k}$ to $10 \mathrm{kG}$, which indicates that the MRE has superparamagnetism and zero coercivity at room temperature; this means that magnetism is completely attributed to the external magnetic field. Moreover, as we expected, SEBS did not change in the presence of the magnetic field. To test the mechanical property, the uni-axial tensile experiments were performed on the prepared MRE films with different component ratios of SEBS powder to liquid paraffin to magnetic fluid as shown in Figure 1f. As the applied strain increases, the stress rises accordingly in a proportional relationship. The stress gradually decreases as the liquid paraffin increases under the same strain condition. The best toughness was achieved at SEBS: liquid paraffin: magnetic fluid =1:3:1, which could reach $400 \%$ strain, and the corresponding stress was $100 \mathrm{KPa}$. The actual stretching is shown in the illustration of Figure 1f, which indicates that MRE film has excellent tensile properties. In order to deliver a larger stretching range and better toughness, a 1:3:1 blending ratio of SEBS powder, to liquid paraffin to magnetic fluid was chosen for this experiment to make MRE films.

Figure 2a shows the electron cloud and potential energy profile diagram [40]. PTFE is used as a negative triboelectric electric material to build a triboelectric pair with $\mathrm{Al}$, which also works as a single electrode. Until the atoms of PTFE and Al materials come into contact, their electron clouds remain separate (Figure 2a(i)). When PTFE and Al are close together within a magnetic field, the electron clouds of the atoms of the two materials overlap, forming ionic or covalent bonds. Then, electrons can be transferred from one atom to another (Figure 2a(ii)). When PTFE is separated from Al, the transferred electrons are still present on the material surface as electrostatic charges (Figure $2 a(i i i)$ ). Figure $2 b$ shows the working mechanism of the device. When the magnet is away from the TENG device, the MRE film is not deformed and the gap between the PTFE and Al films is stable. The charges on the surfaces of the PTFE and Al films are balanced, which does not cause a change in the open-circuit voltage (Figure $2 b(i)$ ). When the magnet slowly approaches the TENG device, the deformation of the MRE brings the PTFE film closer to the $\mathrm{Al} \mathrm{film} \mathrm{(Figure} 2 \mathrm{~b}$ (ii,iii)). The deformation of the MRE film causes an electrical signal to be outputed by the TENG. The surface charge density between PTFE and Al is set to $1 \times 10^{-5} \mathrm{C} \cdot \mathrm{m}^{-2}$ by COMSOL software simulation. As illustrated in Figure 2c, when 
MRE film transitions from the unbending state (i) to the intermediate state (ii) and then to the state with the maximum bending (iii), the potential difference between PTFE and Al interface becomes larger and larger as the separation distance increases, which drives the electrons to flow to the ground. Furthermore, when a gradually increasing magnetic field is applied to the TENG, the maximum force achieved by the mechanical simulation of the MRE is $70 \mathrm{kN} / \mathrm{m}^{2}$, as shown in Figure $\mathrm{S} 1$.

a

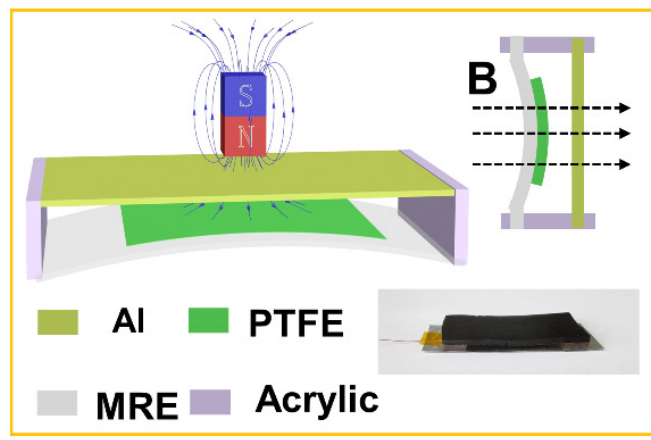

b
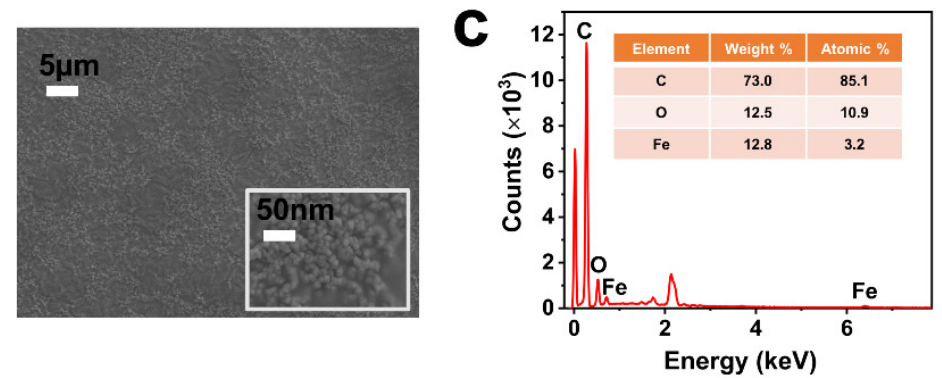

f

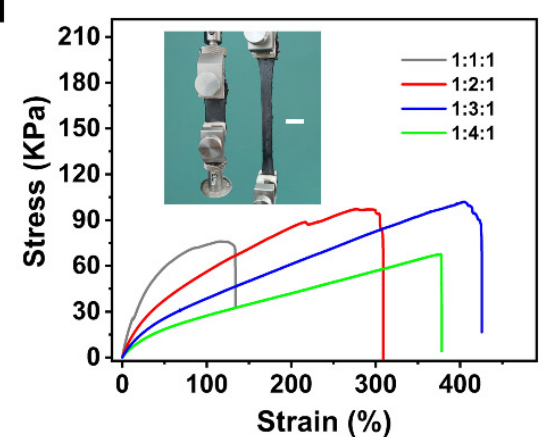

Figure 1. Device models and performance characterization. (a) Diagram of device and working model; (b) SEM images, (c) EDS and (d) XRD of the MRE film; (e) The hysteresis loop of the SEBS and MRE; (f) Stress and strain analysis of the MRE film in different proportions, and photographs of the actual stretching (scale bar is $2 \mathrm{~cm}$ ).

In order to accurately test the electrical properties of the MRE-based TENG, a programmable linear motor was used. As shown in Figure $3 a, b$, the open-circuit voltage and short-circuit current are $30 \mathrm{~V}$ and $0.18 \mu \mathrm{A}$, respectively. In addition, the increase in the load resistance causes the voltage to increase, and the power tends to increase and then decrease, reaching a maximum of $1.3 \mu \mathrm{W}$ at $80 \mathrm{M} \Omega$, as depicted in Figure 3c. It means that the internal resistance of the TENG is about $80 \mathrm{M} \Omega$, which provides the maximum output power in the external circuit when the load resistance is approximately equal to the internal resistance. Here, we changed the magnetic field strength and the frequency of the magnetic field applied to the TENG to observe the voltage output. As shown in Figure $3 \mathrm{~d}$ and Figure S2, the higher the magnetic field strength, the higher the voltage output for the same frequency. With the rising frequency, the voltage also increases for the same magnetic field strength. 


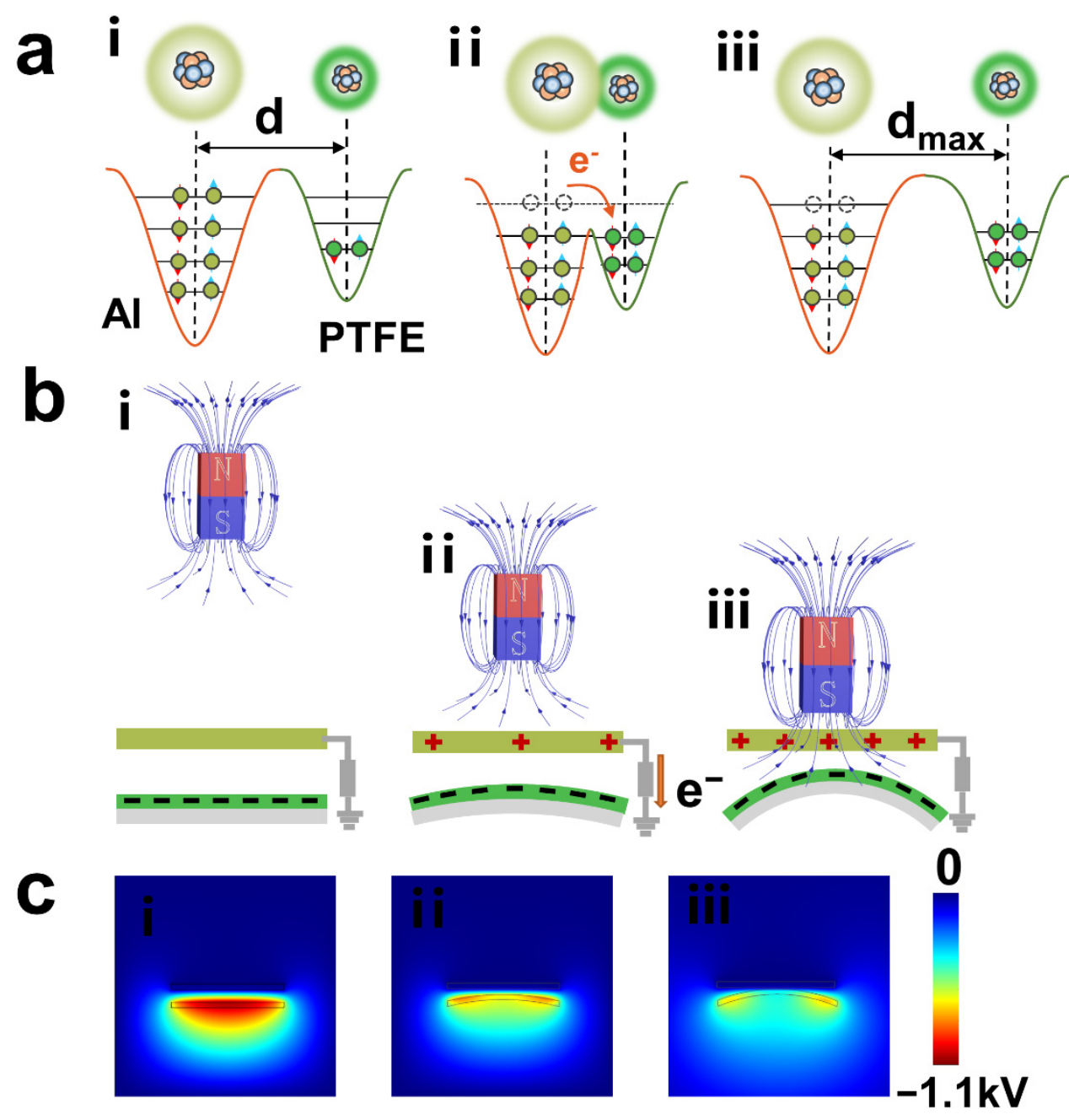

Figure 2. The working mechanism of the triboelectric nanogenerator. (a) Schematic of the electron cloud and potential energy profile of two atoms belonging to PTFE and Al, respectively; (b) A diagram of the working schematic; (c) Simulating the electric potential distribution diagram with COMSOL.

After previous tests, we have learned about the output signal characteristics of the MRE-based TENG affected by magnetic fields. Based on the LabVIEW program, a pointertype magnetic field sensor was designed based on the TENG. The schematic diagram and physical picture are shown in the inset of Figure 4a, which uses an acrylic plate as the base of the cylinder, with $\mathrm{Al}$ electrodes symmetrically pasted on all four sides to form 4 channels and numbered. A long cylindrical MRE with multiple PTFE films is fixed in the middle of the cylinder as a pointer. Figure $4 \mathrm{a}, \mathrm{b}$ plots the voltage output when the magnet is close to channel 1 in 3D and 2D, both of which clearly show a significant rise in voltage peaks and have a fast response of $80 \mathrm{~ms}$. To judge the magnetic field strength by the magnitude of the electrical peak, the output voltage of the device under different magnetic field strengths is illustrated in Figure 4c. The output voltage grows slowly and shows a desirable sensitivity of $31.6 \mathrm{mV} / \mathrm{mT}$ when the magnetic field strength is less than $60 \mathrm{mT}$, while the output voltage increases significantly with a magnetic field strength greater than $60 \mathrm{mT}$. Durability is another critical factor for the sensor. During 2000 cycles of contact-separation cycles, the voltage reveals a negligible change, as depicted in Figure S3. All of these results suggest that the magnetic sensor is robust enough to work normally under various environmental conditions. To better demonstrate the monitoring capability of the sensor, we performed the test shown in Figure 4d and Figure S4. When a magnetic field is applied in one direction, the corresponding direction channel light turns red, while the other channel's lights remain 
green, and the voltage value for each channel is shown in the interface. This result shows that the sensor we designed exhibits excellent real-time magnetic field monitoring and a unique self-powered capability. It also shows that the MRE-based TENG has great potential for magnetic field detection and other smart applications.
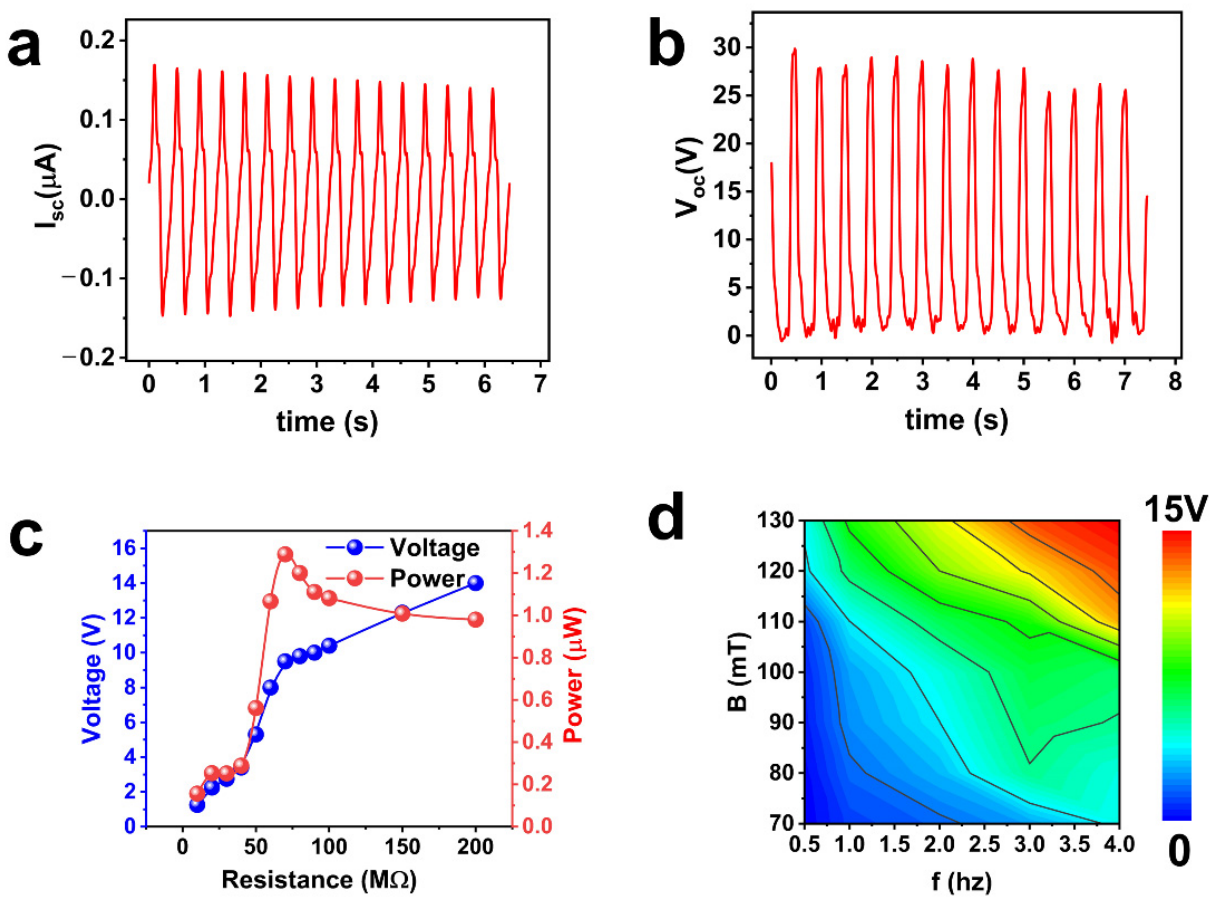

Figure 3. The output performance of TENG. $(\mathbf{a}, \mathbf{b})$ Open-circuit voltage and short-circuit current at the sliding velocity of $16 \mathrm{~cm} \cdot \mathrm{s}^{-1}$; (c) Voltage and power under the different external load resistances; (d) The output performance of the TENG under different sliding frequencies and magnetic field strengths.
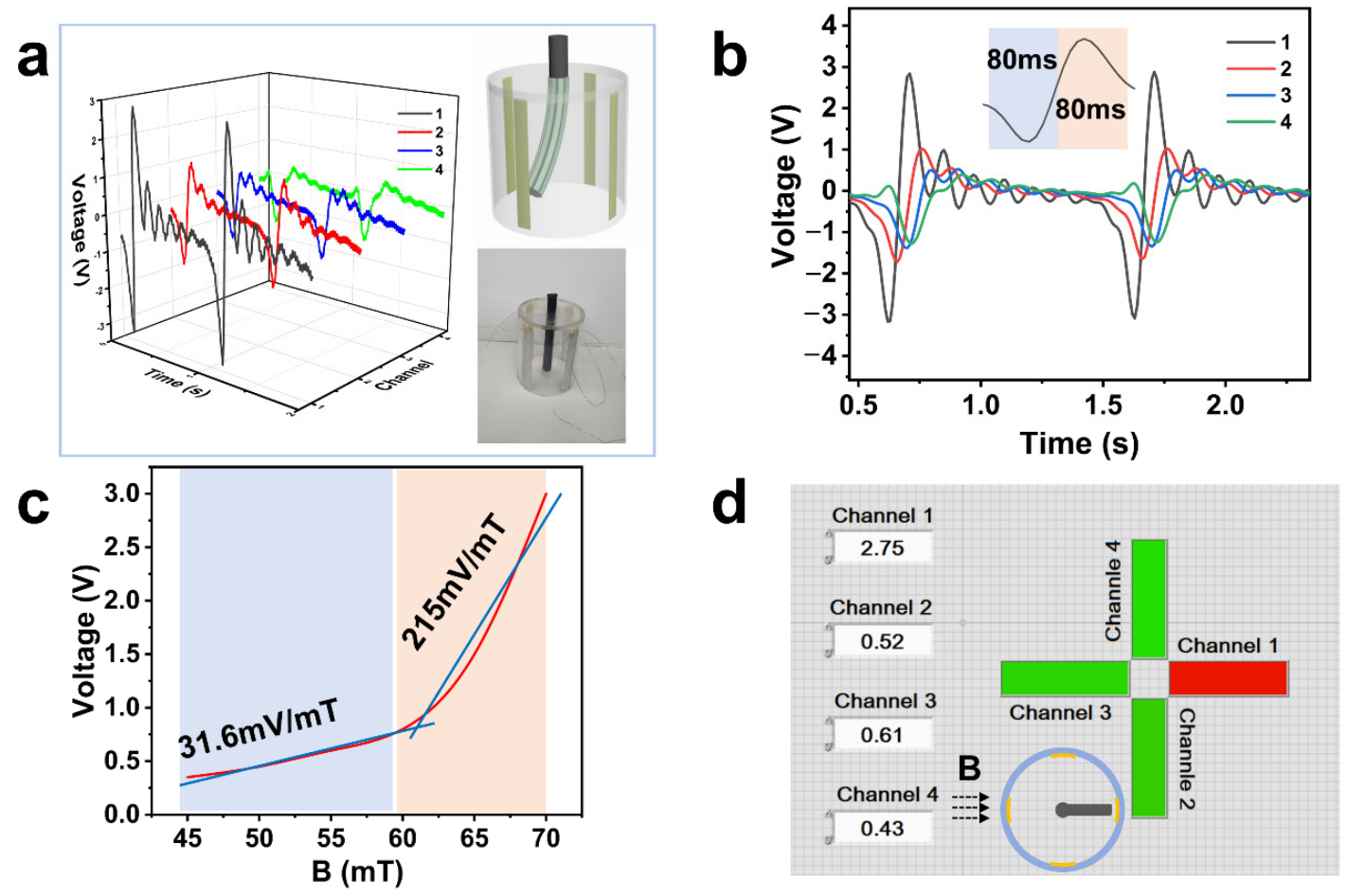

Figure 4. Magnetic field monitoring applications. Measured voltage signals of the sensor when the magnet is close to 1 in 3D (a) and 2D (b),inset is Sensormodel; (c) The output performance of a single channel of the sensor under different magnetic field strengths; (d) Mapping image when the magnet is close to channel 1. 


\section{Conclusions}

In summary, we invented a self-powered magnetic field monitoring sensor consisting of the MRE-based TENG. MRE film is made by mixing SEBS powder with liquid paraffin and magnetic fluid in a certain mass ratio and then heating at high temperature to make an MRE film. Simulating the magnetic field environment, the MRE-based TENG exhibits good output performance with an open-circuit voltage and short-circuit current of $16 \mathrm{~V}$ and $0.18 \mu \mathrm{A}$, respectively, and a maximum output power of $1.3 \mu \mathrm{W}$ at $80 \mathrm{M} \Omega$. Finally, with the assistance of a programmable platform, the pointer-based TENG structure was designed to achieve excellent real-time magnetic field monitoring and a unique self-powered capability. The fabricated magnetic sensor shows a fast response of $80 \mathrm{~ms}$ and a desirable sensitivity of $31.6 \mathrm{mV} / \mathrm{mT}$ in a magnetic field range of $35-60 \mathrm{mT}$ as well as the preliminary vectorability enabled by the multichannel layout. Our work provides a new route of magnetic field measurements and further pushes the application of triboelectric technology in future sensing.

\section{Experimental Section}

Preparation of MRE film: Firstly, SEBS powder (TSRC Nantong Industrial Co., Ltd., Nantong, China) was mixed with liquid paraffin (Shangqiu Liangfeng Health Care Co., Ltd., Shangqiu, China) and magnetic fluid (Ink king magnetic nanofluid company, Jiaxing, China) in a certain mass ratio and mixed in a beaker to obtain a homogeneous mixture. A certain amount of the mixture was put into a porcelain square with a length, width, and height of $60 \mathrm{~mm}, 30 \mathrm{~mm}$, and $20 \mathrm{~mm}$, respectively. Then, the porcelain ark was placed in a high-temperature resistance furnace (Shanghai Boxun Industrial Co., Ltd., Shanghai, China) and heated to $225^{\circ} \mathrm{C}$ or $30 \mathrm{~min}$. Finally, the porcelain ark was left at room temperature until the molten was cooled, solidified, and peeled from the porcelain ark carrier to obtain the MRE film.

Production of sensor: we used an acrylic cylinder with a diameter of $45 \mathrm{~mm}$ and a height of $60 \mathrm{~mm}$ as the housing for the magnetic field monitoring sensor. A cylinder MRE with a diameter of $7 \mathrm{~mm}$ and a height of $55 \mathrm{~mm}$ was fixed in the middle of the cylinder on top of the housing. Four $55 \times 10 \mathrm{~mm} \mathrm{Al} \mathrm{sheets,} \mathrm{which} \mathrm{serve} \mathrm{as} \mathrm{positive} \mathrm{friction} \mathrm{material}$ and electrodes, were evenly attached to the inner wall of the housing as four channels. Multiple pieces of $1 \mathrm{~mm} \times 55 \mathrm{~mm}$ PTFE films were attached around the cylindrical MRE as the negative friction material.

Output performance measurements: The MRE morphology was characterized by the SEM technique (Hitachi SU8020, Tokyo, Japan). Mechanical analysis was performed using a dynamic mechanical analyzer (Mark-10 Corporation, Copiague, NY, USA) based on an M5-20 dynamometer. In addition, all electrical measurements were performed through a programmable Labview platform consisting of a Keithley 6514, Stanford SR570, and a data acquisition module.

Supplementary Materials: The following are available online at https:/ /www.mdpi.com/article/10 $.3390 /$ nano11112815/s1, Figure S1: Simulation drawing of stress and strain experiment, Figure S2: The output performance of the TENG under different sliding frequencies and magnetic field strengths in 2D, Figure S3: The cycle tests of the TENG devices, Figure S4: Mapping image when the magnet is close to channels 2, 3 and 4, Table S1: Comparison of the achievement of our sensor with other competing sensing technologies.

Author Contributions: Conceptualization, H.Z. and K.Z.; methodology, H.Z.; software, N.M. and Z.W.; validation, H.Z. and K.Z.; formal analysis, Z.W. and X.C.; investigation, D.W.; resources, H.Z.; data curation, T.Z. and X.C.; writing—original draft preparation, D.W., N.M. and T.Z.; writingreview and editing, H.Z. and K.Z.; visualization, K.Z.; supervision, H.Z. and K.Z.; project administration, K.Z.; funding acquisition, H.Z. All authors have read and agreed to the published version of the manuscript.

Funding: This research was funded by Shanxi Scholarship Council of China, grant number HGKY2019022 and Youth Top Talent Program of Shanxi Province (2018). 
Conflicts of Interest: The authors declare no conflict of interest.

\section{References}

1. Mishra, S.R.; Dickey, M.D.; Velev, O.D.; Tracy, J.B. Selective and directional actuation of elastomer films using chained magnetic nanoparticles. Nanoscale 2016, 8, 1309-1313. [CrossRef]

2. Su, Y.; Qiu, T.; Song, W.; Han, X.; Sun, M.; Wang, Z.; Xie, H.; Dong, M.; Chen, M. Melt Electrospinning Writing of Magnetic Microrobots. Adv. Sci. 2021, 8, 2003177. [CrossRef]

3. Schmauch, M.M.; Mishra, S.R.; Evans, B.A.; Velev, O.D.; Tracy, J.B. Chained Iron Microparticles for Directionally Controlled Actuation of Soft Robots. ACS Appl. Mater. Interfaces 2017, 9, 11895-11901. [CrossRef] [PubMed]

4. $\mathrm{Hu}, \mathrm{X} . ;$ Nian, G.; Liang, X.; Wu, L.; Yin, T.; Lu, H.; Qu, S.; Yang, W. Adhesive Tough Magnetic Hydrogels with High Fe $\mathrm{H}_{4}$ Content. ACS Appl. Mater. Interfaces 2019, 11, 10292-10300. [CrossRef] [PubMed]

5. Feng, J.; Xuan, S.; Ding, L.; Gong, X. Magnetoactive elastomer/PVDF composite film based magnetically controllable actuator with real-time deformation feedback property. Compos. Part A Appl. Sci. Manuf. 2017, 103, 25-34. [CrossRef]

6. Gao, W.; Wang, L.; Wang, X.; Liu, H. Magnetic Driving Flowerlike Soft Platform: Biomimetic Fabrication and External Regulation. ACS Appl. Mater. Interfaces 2016, 8, 14182-14189. [CrossRef]

7. Li, C.; Lau, G.C.; Yuan, H.; Aggarwal, A.; Dominguez, V.L.; Liu, S.; Sai, H.; Palmer, L.C.; Sather, N.A.; Pearson, T.J.; et al. Fast and programmable locomotion of hydrogel-metal hybrids under light and magnetic fields. Sci. Robot. 2020, 5, eabb9822. [CrossRef]

8. Stanier, D.C.; Ciambella, J.; Rahatekar, S.S. Fabrication and characterisation of short fibre reinforced elastomer composites for bending and twisting magnetic actuation. Compos. Part A Appl. Sci. Manuf. 2016, 91, 168-176. [CrossRef]

9. Hajra, S.; Vivekananthan, V.; Sahu, M.; Khandelwal, G.; Joseph Raj, N.P.M.; Kim, S.J. Triboelectric nanogenerator using multiferroic materials: An approach for energy harvesting and self-powered magnetic field detection. Nano Energy 2021, 85, 105964. [CrossRef]

10. Fan, D.; Wang, Q.; Zhu, T.; Wang, H.; Liu, B.; Wang, Y.; Liu, Z.; Liu, X.; Fan, D.; Wang, X. Recent Advances of Magnetic Nanomaterials in Bone Tissue Repair. Front. Chem. 2020, 8, 745. [CrossRef]

11. Stepanov, R.A.; Sokoloff, D.D. Magnetic helicity and prospects for its observation in the interstellar medium. Physics-Uspekhi 2019, 62, 1208. [CrossRef]

12. Silveyra, J.M.; Ferrara, E.; Huber, D.L.; Monson, T.C. Soft magnetic materials for a sustainable and electrified world. Science 2018, 362. [CrossRef] [PubMed]

13. Su, S.; Li, H.; Huang, J.; Zhang, Z.; Liang, C.; Jiang, W.; Deng, A.; Liu, K.; Shi, Z.; Qian, D.; et al. Patterning Graphene Films by H2O-Based Magnetic-Assisted UV Photolysis. ACS Appl. Mater. Interfaces 2020, 12, 55382-55389. [CrossRef] [PubMed]

14. Shalaby, M.; Shokair, M.; Messiha, N.W. Electromagnetic Field Measurement Instruments: Survey. Iran. J. Sci. Technol. Trans. Electr. Eng. 2018, 43, 1-14. [CrossRef]

15. Ni, J.L.; Hu, F.; Feng, S.J.; Kan, X.C.; Han, Y.Y.; Liu, X.S. Soft magnetic properties of FeSiAl/carbonyl iron composites with high magnetic permeability and low magnetic loss. J. Alloys Compd. 2021, 887, 161337. [CrossRef]

16. Wang, S.; Yuan, F.; Liu, S.; Zhou, J.; Xuan, S.; Wang, Y.; Gong, X. A smart triboelectric nanogenerator with tunable rheological and electrical performance for self-powered multi-sensors. J. Mater. Chem. C 2020, 8, 3715-3723. [CrossRef]

17. Deng, H.; Sattari, K.; Xie, Y.; Liao, P.; Yan, Z.; Lin, J. Laser reprogramming magnetic anisotropy in soft composites for reconfigurable 3D shaping. Nat. Commun. 2020, 11, 6325. [CrossRef]

18. Zhu, M.; Qi, S.; Xie, Y.; Fu, J.; Yu, M. Transient responses of magnetorheological elastomer and isolator under shear mode. Smart Mater. Struct. 2019, 28, 044002. [CrossRef]

19. Davis, L.C. Model of magnetorheological elastomers. J. Appl. Phys. 1999, 85, 3348. [CrossRef]

20. Gong, X.L.; Zhang, X.Z.; Zhang, P.Q. Fabrication and characterization of isotropic magnetorheological elastomers. Polym. Test. 2005, 24, 669-676. [CrossRef]

21. Li, W.H.; Zhang, X.Z. A study of the magnetorheological effect of bimodal particle based magnetorheologicalelastomers. Smart Mater. Struct. 2010, 19, 035002. [CrossRef]

22. Wang, S.; He, M.; Weng, B.; Gan, L.; Zhao, Y.; Li, N.; Xie, Y. Stretchable and Wearable Triboelectric Nanogenerator Based on Kinesio Tape for Self-Powered Human Motion Sensing. Nanomaterials 2018, 8, 657. [CrossRef] [PubMed]

23. Zhang, H.; Yang, Y.; Hou, T.-C.; Su, Y.; Hu, C.; Wang, Z.L. Triboelectric nanogenerator built inside clothes for self-powered glucose biosensors. Nano Energy 2013, 2, 1019-1024. [CrossRef]

24. Guo, H.; Pu, X.; Chen, J.; Meng, Y.; Yeh, M.H.; Liu, G.; Tang, Q.; Chen, B.; Liu, D.; Qi, S.; et al. A highly sensitive, self-powered triboelectric auditory sensor for social robotics and hearing AIDS. Sci. Robot. 2018, 3, eaat2516. [CrossRef]

25. Cui, X.; Zhao, T.; Yang, S.; Xie, G.; Zhang, Z.; Zhang, Y.; Sang, S.; Lin, Z.-H.; Zhang, W.; Zhang, H. A spongy electrode-brushstructured dual-mode triboelectric nanogenerator for harvesting mechanical energy and self-powered trajectory tracking. Nano Energy 2020, 78, 105381. [CrossRef]

26. Ren, X.; Fan, H.; Zhao, Y.; Liu, Z. Flexible Lead-Free BiFeO3/PDMS-Based Nanogenerator as Piezoelectric Energy Harvester. ACS Appl. Mater. Interfaces 2016, 8, 26190-26197. [CrossRef]

27. Chen, C.; Guo, H.; Chen, L.; Wang, Y.C.; Pu, X.; Yu, W.; Wang, F.; Du, Z.; Wang, Z.L. Direct Current Fabric Triboelectric Nanogenerator for Biomotion Energy Harvesting. ACS Nano 2020, 14, 4585-4594. [CrossRef] [PubMed]

28. Chen, J.; Wang, Z.L. Reviving Vibration Energy Harvesting and Self-Powered Sensing by a Triboelectric Nanogenerator. Joule 2017, 1, 480-521. [CrossRef] 
29. Wan, D.; Yang, J.; Cui, X.; Ma, N.; Wang, Z.; Li, Y.; Li, P.; Zhang, Y.; Lin, Z.; Sang, S.; et al. Human body-based self-powered wearable electronics for promoting wound healing driven by biomechanical motions. Nano Energy 2021, 89, 106465. [CrossRef]

30. Maharjan, P.; Bhatta, T.; Cho, H.; Hui, X.; Park, C.; Yoon, S.; Salauddin, M.; Rahman, M.T.; Rana, S.S.; Park, J.Y. A Fully Functional Universal Self-Chargeable Power Module for Portable/Wearable Electronics and Self-Powered IoT Applications. Adv. Energy Mater. 2020, 10, 2002782. [CrossRef]

31. Yang, X.; Chan, S.; Wang, L.; Daoud, W.A. Water tank triboelectric nanogenerator for efficient harvesting of water wave energy over a broad frequency range. Nano Energy 2018, 44, 388-398. [CrossRef]

32. Hajra, S.; Padhan, A.M.; Sahu, M.; Alagarsamy, P.; Lee, K.; Kim, H.J. Lead-free flexible Bismuth Titanate-PDMS composites: A multifunctional colossal dielectric material for hybrid piezo-triboelectric nanogenerator to sustainably power portable electronics. Nano Energy 2021, 89, 106316. [CrossRef]

33. Yang, Y.; Lin, L.; Zhang, Y.; Jing, Q.; Hou, T.-C.; Wang, Z.L. Self-Powered Magnetic Sensor Based on a Triboelectric Nanogenerator. ACS Nano 2012, 6, 10378-10383. [CrossRef]

34. Qi, S.; Guo, H.; Chen, J.; Fu, J.; Hu, C.; Yu, M.; Wang, Z.L. Magnetorheological elastomers enabled high-sensitive self-powered tribo-sensor for magnetic field detection. Nanoscale 2018, 10, 4745-4752. [CrossRef]

35. Cai, Y.W.; Zhang, X.N.; Wang, G.G.; Li, G.Z.; Zhao, D.Q.; Sun, N.; Li, F.; Zhang, H.Y.; Han, J.C.; Yang, Y. A flexible ultra-sensitive triboelectric tactile sensor of wrinkled PDMS/MXene composite films for E-skin. Nano Energy 2021, 81, 105663. [CrossRef]

36. Graham, S.A.; Chandrarathna, S.C.; Patnam, H.; Manchi, P.; Lee, J.W.; Yu, J.S. Harsh environment-tolerant and robust triboelectric nanogenerators for mechanical-energy harvesting, sensing, and energy storage in a smart home. Nano Energy 2021, 80, 105547. [CrossRef]

37. Zhang, T.; Wen, Z.; Liu, Y.; Zhang, Z.; Xie, Y.; Sun, X. Hybridized Nanogenerators for Multifunctional Self-Powered Sensing: Principles, Prototypes, and Perspectives. iScience 2020, 23, 101813. [CrossRef] [PubMed]

38. Lama, J.; Yau, A.; Chen, G.; Sivakumar, A.; Zhao, X.; Chen, J. Textile triboelectric nanogenerators for self-powered biomonitoring. J. Mater. Chem. A 2021, 9, 19149-19178. [CrossRef]

39. Song, X.; Wang, W.; Yang, F.; Wang, G.; Rui, X. The study of enhancement of magnetorheological effect based on natural rubber/thermoplastic elastomer SEBS hybrid matrix. J. Intell. Mater. Syst. Struct. 2020, 31, 339-348. [CrossRef]

40. Wang, Z.L.; Wang, A.C. On the origin of contact-electrification. Mater. Today 2019, 30, 34-51. [CrossRef] 